\title{
Design and Development of Size Segregation Sieve Machine with Centrifugal Action
}

\author{
Man Djun Lee*, Pui San Lee \\ School of Engineering and Technology, University College of Technology Sarawak, 96000, Sarawak, Malaysia
}

Received March 11, 2020; Revised May 23, 2020; Accepted June 4, 2020

Copyright $(2020$ by authors, all rights reserved. Authors agree that this article remains permanently open access under the terms of the Creative Commons Attribution License 4.0 International License

\begin{abstract}
This study aims to design and develop a size segregation sieve machine that utilizes centrifugal action to separate impurities from short Oil Palm Frond (OPF) fibers. OPF fibers can manufacture into various products such as erosion control mat and medium density fibreboard as an agricultural waste management program for achieving environmental sustainability. In this aspect, the role of the fiber sieving machine would be significant to support the industry in the production of OPF fibers. However, a thorough literature survey reviewed that limited publications are available in this area; most work done is published in the form of patents. In addition, most fiber sieving machines available in the market utilize manual labour work in the separation phase, where they are highly inefficient. Therefore, this study is designed to fill these gaps. The design of this study adopts the mechanism of various size segregation concepts available and includes centrifugal action in the separation process to increase efficiency. Eventually, a prototype was fabricated for laboratory testing. Several vital parameters are highlighted, which includes mesh surface inclination, sieving duration, a rotation speed of screen and maximum capacity that significantly affects the sieving efficiency. Findings from this study show that sieving duration is less significant to the developed design as higher rotation speed will tend to improve the passing percentage of the fibers. Testing results also revealed the potential application of such a machine in other particle separation applications such as soil separation. For future study, it is recommended to improve the current design in terms of parts, size simplification and multiple size segregation adaptation for achieving a higher production rate.
\end{abstract}

Keywords Oil Palm Frond Fibers, Waste Management, Fiber Separation, Machine Design, Soil Separation

\section{Introduction}

Malaysia is currently the world's second-largest producer of palm oil after Indonesia, with $39 \%$ of global production (Liew, Kassim, Muda, Loh, \& Affam, 2014). The issue of environmental threat in the country due to the relevant economic activities has received considerable critical attention (Lee, Osman, Wong, \& Ngu, 2018; Sukiran, Abnisa, Wan Daud, Abu Bakar, \& Loh, 2017; Zainal, Jalani, Mamat, \& Astimar, 2017). Despite the product of oil palm has significant value, the oil palm wastes include oil palm trunks (OPT), oil palm fronds (OPF) and empty fruit bunches (EFB) has created disposal problem (Abdullah, 2013). OPT usually is left to burn or decay in the plantation area which both have disadvantages due to the high moisture content of OPT meaning that it cannot be easily burned and also decomposing relatively take a long time to complete which is five years (Rosli et al., 2016). The fronds can be processed into various useful materials (Sukiran et al., 2017). The first method is by using the chemical to treat the raw input, and the alternative way is by using physical breakdown (Sandberg, Kutnar, \& Mantanis, 2017). In this case, the raw input undergoes shredding, pressing or crushing and lastly sieving. The shredded residue contains large pieces called pulp and cellulose fibre. The pulp and fibre also have various uses such as fertilizer and in mattress production (Colombo \& Vergani, 2018). Before sieving, the shredded residue needs to be broke into a smaller fraction by either crusher or hammer mill. Then the residue impurity needs to be sieved from fibre before production. The sieved fibre stack can be sold to manufacturers for many other applications.

Sieving process is the process to separate larger particles from a mixture (Liu, 2009). While sieve is a piece of equipment that has holes punched in it or made of crossed wires for the use of separating larger particles from a mixture. The size of particles allowed to pass through depends on the sieve sizes which is the area of a single hole on the sieve screen, while the trapped particles are usually the unwanted residue in which they are too large to pass through the holes. Sieve is generally used in many fields such as medicine, gardening, cooking, building construction, industry, and it also uses to separate fibre. 
The traditional sieving method is hand sieving, due to its non-consistency, low accuracy and precision before the development of modern sieve that is more practical. However, there is no much attention paid to the research about sieve (Leschonski, 1979). The introduction of machine sieve significantly reduces the users' workload as well as time consumption because the machine takes over the repetition of the sieving process with high precision, unlike the uncontrollable traditional method variables. In the present, there are many sieving mechanisms that can be referred from other agriculture products such as wind sieve used in separation of seed and shells as well as vibrating screen and centrifugal sieve. These mechanisms or methods are more likely to serve for size separation, which is the primary concern of this study. In this study, the goal is to create a device that could separate impurity from fibers using appropriate techniques that have better production efficiency. The conventional design creates vibration forces in a radial direction, which causes the trapped object to accumulate at a specific point and eventually removed from an outlet. In this study, the vibration forces will refer to Coriolis force and centrifugal force that characterized by a rotating flat round screen with significant elevation.

\section{Theoretical Framework for Design Considerations}

\subsection{Rotary Sieve Action}

The key function of the machine sieve is to separate impurity from fibers or vice versa, so a suitable sieve size shall maximize the probability to separate the impurities. The moisture content and water in the fiber will increase the weight of fiber and tend to stick the fiber together in which will reduce the efficiency of some sieve method (Liu, 2009). Therefore, the drying process shall be considered before or after the shredding process to reduce the moisture content. Air drying will be a cheap solution besides using a dryer. In the sieve concept, the mixture shall be distributed evenly on the sieve screen to maximize the sieving efficiency (Liu, 2009). Therefore, the mesh size and shape are among the major concerns. Moreover, the pushing or pulling mechanism, for example vibrating screen is used with the principle of creating vibration forces to enable the smaller particles of a mixture to enter the holes, generally depending on gravity to pull the particles to pass through the holes. Centrifugal sieve is depending on the centrifugal force while wind sifter uses the pressure difference to push the particles. The pulling or pushing forces are sensitive as small force unable to do the work while extra force will break the fibre into an undesirable condition in the worst cases. Rotary sieve is suitable to sort the hard materials such as soil and grain. The forces involved in the material movement in the rotary sieve are gravitational force, centrifugal force and frictional force (Elamin, 1981).

\section{Gravitational force: $m g$}

Where $m=$ mass of material; $g=$ gravitational acceleration.

$$
\text { Centrifugal force: } m r \omega^{2}
$$

Where $m=$ mass of material; $r=$ radius of the sieve; $\omega=$ angular velocity.

Frictional force: $T=\mu N$

Where $\mathrm{T}=$ frictional force; $\mu=$ coefficient of friction; $\mathrm{N}$ $=$ normal force

The assumptions of the rotary sieve:

The cylinder's inner surface is smooth.

The $\mathrm{x}$ and $\mathrm{y}$ axes are revolving together with the cylinder.

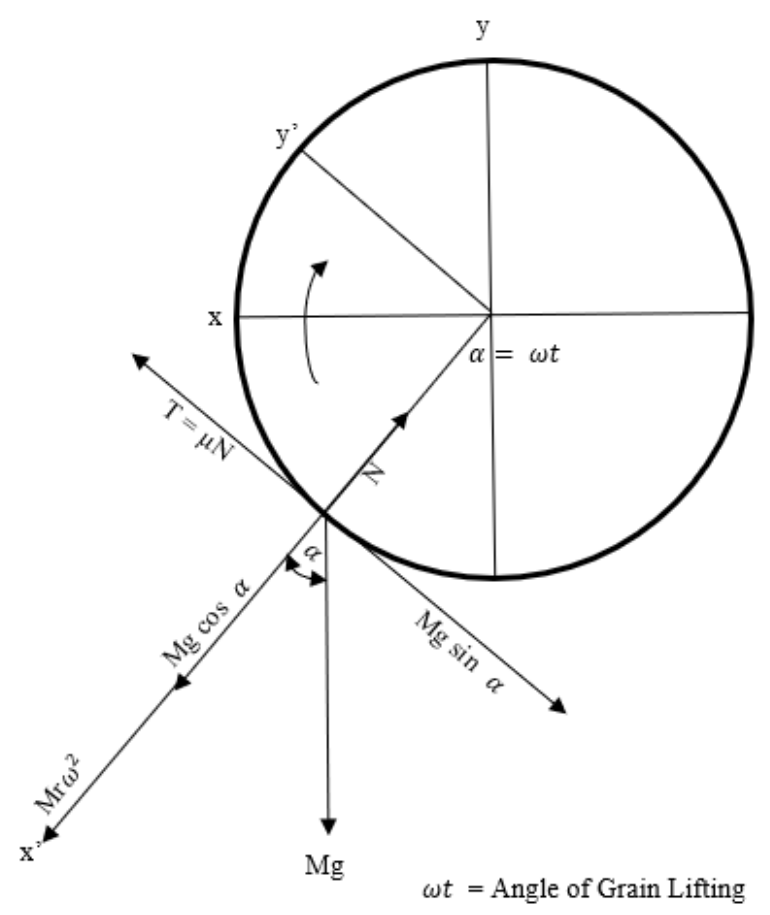

Figure 1. Force Acting on the Materials in Cylinder Sieve (Elamin, 1981)

For materials equilibrium in this moment of time is:

$$
\begin{gathered}
\mathrm{mr} \omega^{2}-\mathrm{N}+\mathrm{mg} \cos \omega \mathrm{t}=0 \\
N=m\left(r \omega^{2}+g \cos \omega t\right)
\end{gathered}
$$

The frictional force is:

$$
T=\mu N=m g \sin \omega t
$$

In this case, the kinematic factor of motion is assumed to be

$$
k=\frac{r \omega^{2}}{g}
$$

From equation 1,

$$
\begin{gathered}
N=m g(k+\cos \omega t) \\
\mu=\tan \phi
\end{gathered}
$$


$\phi$ is the angle of material friction against the cylinder inner surface. Assuming there is no relative velocity of the materials or equal to zero, $N$ and $\mu$ can be substituted into equation 2.

When sliding occurs, the equation will become unbalanced.

$$
\begin{gathered}
\tan \phi m g(k+\cos \omega t) \geq m g \sin \omega t \\
\frac{\sin \phi}{\cos \phi}(k+\cos \omega t) \geq \sin \omega t \\
k+\cos \omega t \geq \frac{\sin \omega t \cos \phi}{\sin \phi} \\
\mathrm{k}=\frac{\sin \omega t \cos \phi}{\sin \phi}-\cos \omega t \\
\sin (\omega t-\phi)=\sin \omega t \cos \phi-\cos \omega t \sin \phi
\end{gathered}
$$

Dividing equation 3 by $\sin \phi$,

$$
\begin{gathered}
k \geq \frac{\sin (\omega t-\phi)}{\sin \phi} \\
k \sin \phi \geq \sin (\omega t-\phi)
\end{gathered}
$$

Denoting the limit angle of grain lifting by

$$
\begin{gathered}
\alpha_{1}=\omega t_{1} \\
\sin \left(\alpha_{1}-\phi\right)=k \sin \phi \\
\alpha_{1}-\phi=\arcsin k \sin \phi \\
\alpha_{1}=\arcsin k \sin \phi+\phi
\end{gathered}
$$

Equation 5 shows that the greater $k$ and $\phi$, the greater the limit angle of lifting. Since $\sin \left(\alpha_{1}-\phi\right) \leq 1$, therefore $k \sin \phi \leq 1$. Hence, the highest value of $\mathrm{k}$ is:

$$
k_{\max }=\frac{1}{\sin \phi}=\sqrt{\frac{\sin ^{2} \phi+\cos ^{2} \phi}{\sin ^{2} \phi}=\sqrt{1+\frac{1}{\mu^{2}}}}
$$

By substituting $\mathrm{k}_{\max }$ into equation 4 ,

$$
\begin{gathered}
\sin \left(\alpha_{1 \max }-\phi\right)=1 \\
\alpha_{1 \max }=\frac{\pi}{2}+\phi
\end{gathered}
$$

Under the influence of gravity, the materials will slide over the internal cylinder surface at the side of the sieve, thus creates a critical angle. Eventually, the materials will cascade down the side of the rotating screen to reach the bottom of the screen. They will slowly roll across the holes in the screen and fall through the screen. The materials will not fall if the RPM of the cylinder is higher than the critical RPM, even under the influence of gravity. At this instant, the materials will rotate together with the sieve, and the critical RPM can be determined by:

$$
\mathrm{n}=\frac{30}{\pi} \sqrt{\frac{\mathrm{kg}}{r}} \mathrm{rpm}
$$

$$
\mathrm{n}=\frac{30}{\pi} \sqrt{\frac{r \omega^{2}}{g} \times \frac{g}{r}}=\frac{30}{\pi} \sqrt{\omega^{2}}=\frac{30}{\pi} \omega
$$

Where $\mathrm{n}=$ number of revolutions per minute; $\mathrm{k}=$ kinematic factor of motion; $\mathrm{g}=$ acceleration due to gravity; $\mathrm{r}=$ radius of the cylinder; $\omega=$ angular velocity

This mathematical framework is significant as it can be applied in most of the spinning sieve machines. In short, to improve the output rate, critical RPM must be achieved. This circumstance also works in most of the spinning mechanisms for the sieving process such as a centrifuge.

\subsection{Incline Surface Angle and Force}

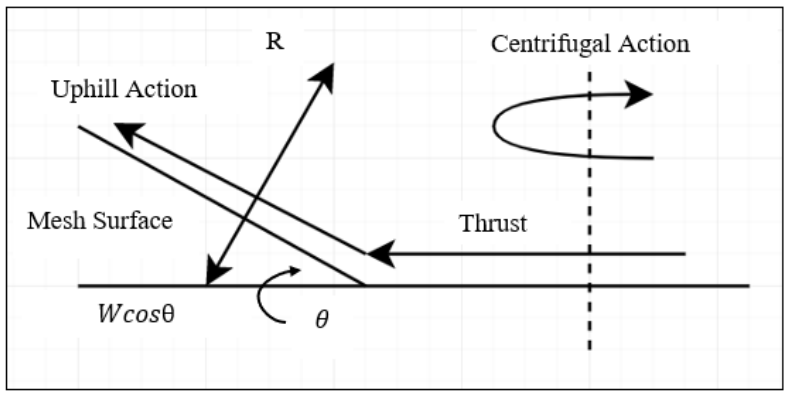

Figure 2. Elevation of Mesh from Horizontal Surface

Based on Figure 2, if subjected to the sieve screen rotation, the objects on the spinning plate will be experiencing centrifugal action. The objects will be pushed outwards and driven towards the incline mesh surface. The angle of the inclined surface would be in the range between $0^{\circ}$ to $90^{\circ}$. In this aspect, the higher the elevation of the angle, the greater the thrust required for the objects to travel to the edge of the mesh surface. For example, an elevation of $48^{\circ}$ will roughly require more $50 \%$ of the thrust than a zero-elevation condition; however, a mesh with perpendicular elevation is impossible to be moved up by the objects. The thrust required can be determined by the following equation:

$$
F_{\text {Required }}=\frac{W_{\text {objects }}+f}{\cos \theta}
$$

$W_{\text {objects }}=$ Weight of the sieved objects

$f=$ Friction of the surface

The thrust that throws the objects in outward action can be increased by higher rotation speed for the centrifugal action. If the inclination is zero, the objects are entirely depending on the downward pulling action due to gravity to pass through the screen.

\subsection{Volume Geometry}

The increasing slope of the materials in the cone container will cause some loss of materials before the small object can pass through the area of the mesh screen. This circumstance will contribute to decreasing sieve efficiency, 
and thus, maximum capacity is the critical parameter to be determined. In this case, the maximum capacity or working volume refers to the amount limitation of the load on the plate, typically computed from cone geometry reduction from a more prominent cone, as shown in Figure 3. The mathematical representation is shown below:

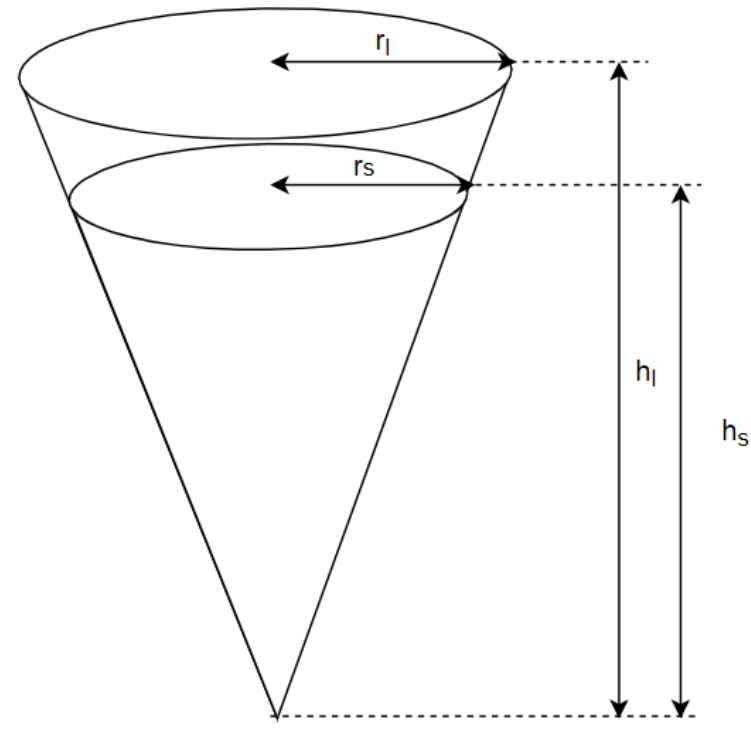

Figure 3. Maximum Volume Geometry

$$
\text { Working volume, } V_{w}=\frac{k \pi\left(r_{l}^{2} h_{l}-r_{s}^{2} h_{s}\right)}{3}
$$

Where $r_{l}=$ Radius of Large Cone;

$r_{s}=$ Radius of Small Cone;

$h_{l}=$ Height of Large Cone;

$h_{s}=$ Height of Small Cone;

$k=$ Critical Ratio Coefficient

The critical ratio coefficient indicates that a certain level of the maximum volume will not show any improvement but throttle the sieving efficiency.

\subsection{Percentage Passing}

Sieve efficiency is typically difficult to be determined as the random motion of particles grants certain probability of passing and losing of the materials in the sieving process. However, it can be observed by knowing the percentage passing and percentage losing of the materials through experiments. The mathematical representation of percentage passing is shown as below:

$$
\text { Percentage of Passing }=\frac{M_{\text {passing }}}{M_{\text {sample }}} \times 100 \%
$$

$\mathrm{M}_{\text {passing }}=$ Actual mass of sample pass through the screen

$\mathrm{M}_{\text {sample }}=$ Total mass of the sample

In this study, the assumption is that only two objects will be segregated. Thus, the total weight of objects can be measured at the beginning. Then after the machine operation, the objects collected from the inner drum or second outlet will be measured its weight for \% passing calculation. The ratio of test samples can calculate the ideal $\%$ passing. The actual $\%$ passing can be compared with the ideal \% passing to show the sieving efficiency of the machine. Higher \% passing means better efficiency.

\subsection{Percentage Losing}

The percentage losing is the opposite of percentage passing, thus higher percentage losing means lower sieve efficiency as some of the objects that should pass through the screen accumulate at the wrong collection tank, which is undesirable. The mathematical representation of percentage losing formula is shown below:

$$
\begin{gathered}
\quad \% \text { Lossing }=\frac{\mathrm{M}_{\text {sample }}-\mathrm{M}_{\text {passing }}}{\mathrm{M}_{\text {sample }}} \times 100 \\
\mathrm{M}_{\text {sample }}=\text { Total mass of the sample } \\
\mathrm{M}_{\text {passing }}=\text { Actual mass of sample pass through the screen }
\end{gathered}
$$

\section{Methodology}

This study started by developing a suitable design concept for the machine. A literature review has been the basis of this study to highlight the design considerations and theoretical framework to generate a conceptual design. The conceptual design is then being developed and tested in the next phase. Several tests were conducted to obtain machine sieve efficiency data. The data are analyzed to highlight the overall performance and limitations of the developed machine.

\subsection{Conceptual Design}

The concept of the design utilizes centrifugal action to push the objects through the sieve screen and the separated objects will free fall to an inclined surface. The conceptual design is shown in Figure 4. In this design, the sieve was made into a conical shape with a flat bottom. The yellow highlighted region in Figure 5 is wire mesh while the flat bottom is made with a steel plate. The plate is installed on a rotating shaft of a motor so that the plate is driven by a rotating force to gain centrifugal action. Initially, the objects on the plate are stationary (Figure 6), when the motor starts spinning, the energy gain on the objects will bring the objects to outward action, causing the objects to travel up the incline cone surface. At that instance, the smaller particles will fall into the mesh holes while the large particles continue to travel to the edge of the mesh, as shown in Figure 7. Eventually, both small and large particles fall into different drums; the larger particles will always fall into the outer drum, then come out from the drum outlet as the red arrows shown in Figure 8. As the bottom surface of the drum is inclined, the accumulated particles will then slide to the outlet region. The schematic 
diagram of how the sieve machine works is shown in Figure 9.

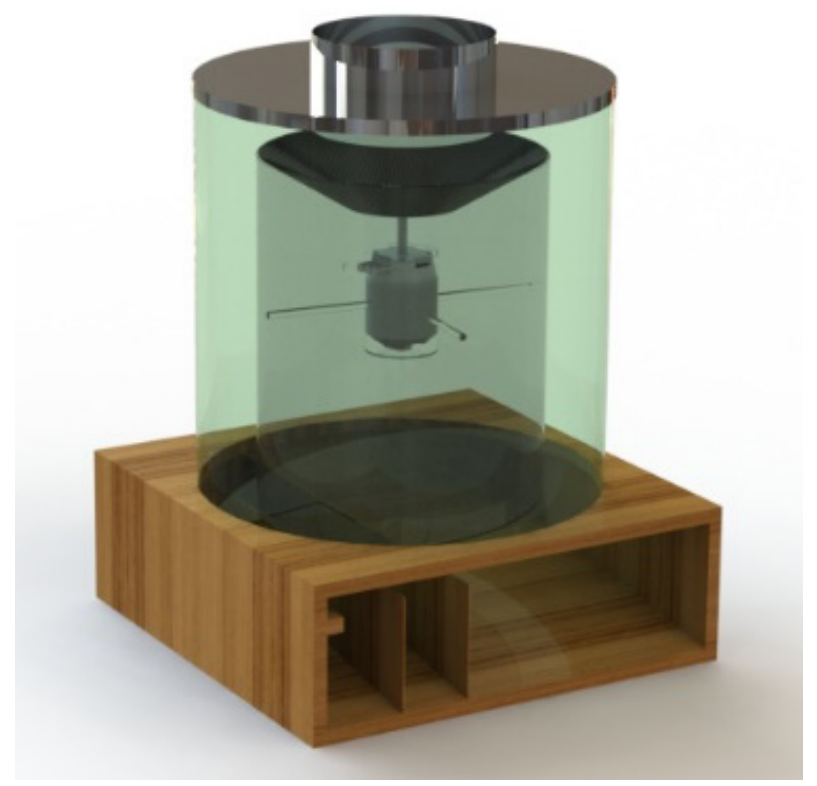

Figure 4. Conceptual Design
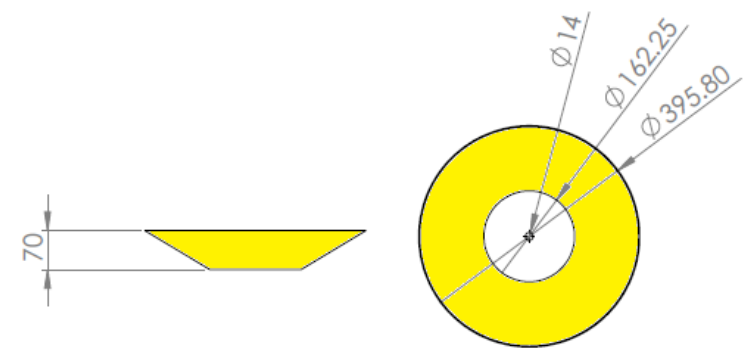

Figure 5. Sieve Screen Design (Measurements in millimeters)

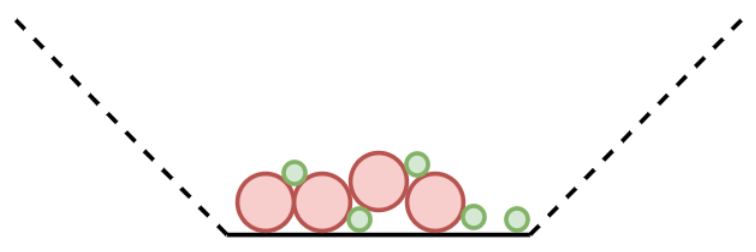

Figure 6. Objects in Stationary Condition

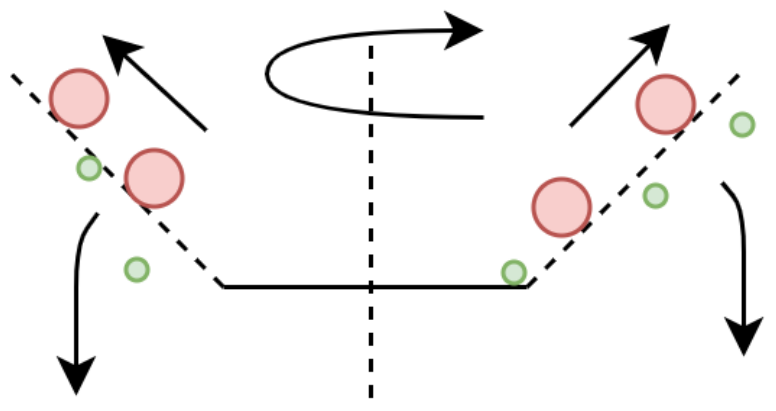

Figure 7. Objects segregated by Centrifuge

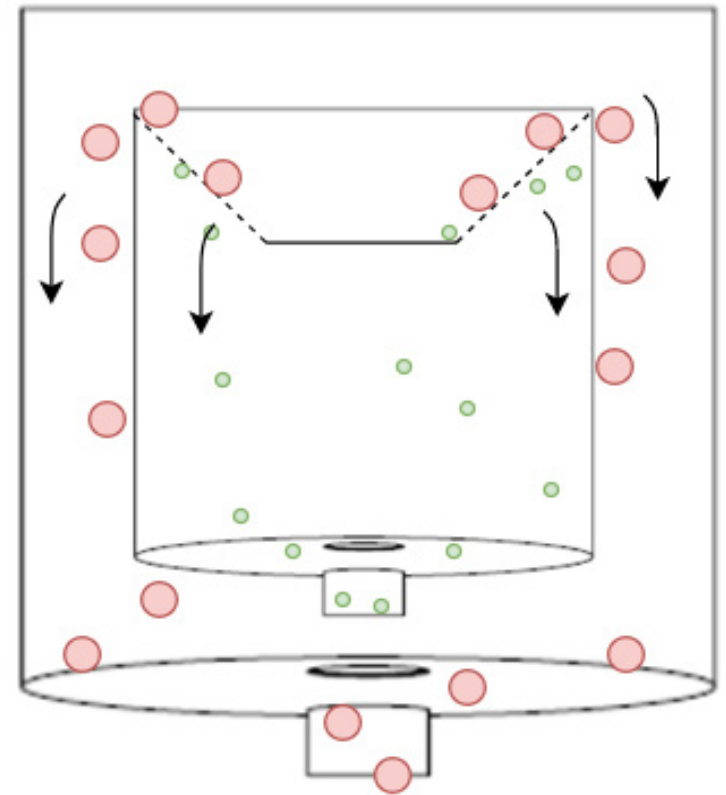

Figure 8. Movement of Particles inside the Drum

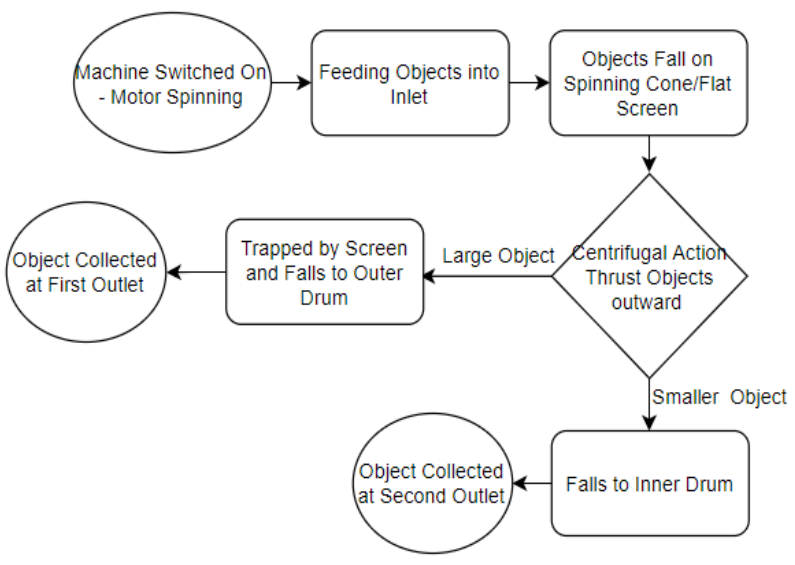

Figure 9. Process Flow of Conceptual Design

\subsection{Design of Experiments}

The conceptual model is developed and tested. The primary concern of the sieve machine is sieve efficiency, which is about percentage passing and percentage losses of the materials. The power consumption data are nonetheless essential to know the overall efficiency of the machine. In this study, the sieving efficiency is recorded in three different categories: sieve efficiency over time, sieve efficiency against total mass, sieve efficiency over RPM. The test samples used in the test are small stainless-steel ball bearings with two different sizes used which would be easy to notice and record, the smaller ball bearings have a mass of $10 \mathrm{~g}$, while larger ball bearings are impossible to pass through sieve screen and have a mass of $16 \mathrm{~g}$. The purpose of the tests is to prove the potential of the developed machine. Moreover, the limitations of the machine could be noticed during the experiments and discussed for future improvement in the design. 


\section{Results and Discussions}

\subsection{Sieve Efficiency over Time}

The data recorded with constant total and sample mass, under 200 RPM, with several attempts to obtain average results. In theory, the sieving process takes place very fast. Thus, the sieving efficiency shall be less dependent on time. The result is presented in Figure 10.

Based on Figure 10, the percentage passing is increasing over time but shows low or zero variance since $6^{\text {th }}$ second for all attempts, this is due to all the samples materials eventually dispensed at that instantaneous of time. It also shows a slow start as the percentage passing is 0 in the first few seconds due to the motor required time for acceleration from the stationary condition to achieve a stable working state. The average correlation of the data is 0.96 , considered almost perfect and shows a positive linear relationship. However, this relationship only takes count after a slow start and before samples dispensed. Even with some elevation of the mesh screen, the sieving efficiency is proven the same relationship, but the percentage passing of the materials is slightly lower under the same RPM.

\subsection{Effects of Rotation Speed}

Ideally, higher RPM usually means higher thrust from centrifugal action. Low thrust will cause the materials to travel insufficient distance before they reach the edge of the mesh surface. Thus, the increase of thrust and uphill action will improve the percentage passing, but there is a cap for zero elevation mesh surface. The problem with flat mesh surface is that a higher amount of material bypasses the screen sieving action as they are thrown at an exceeding thrust. The graph of passing and losing percentage against RPM is shown in Figure 11.

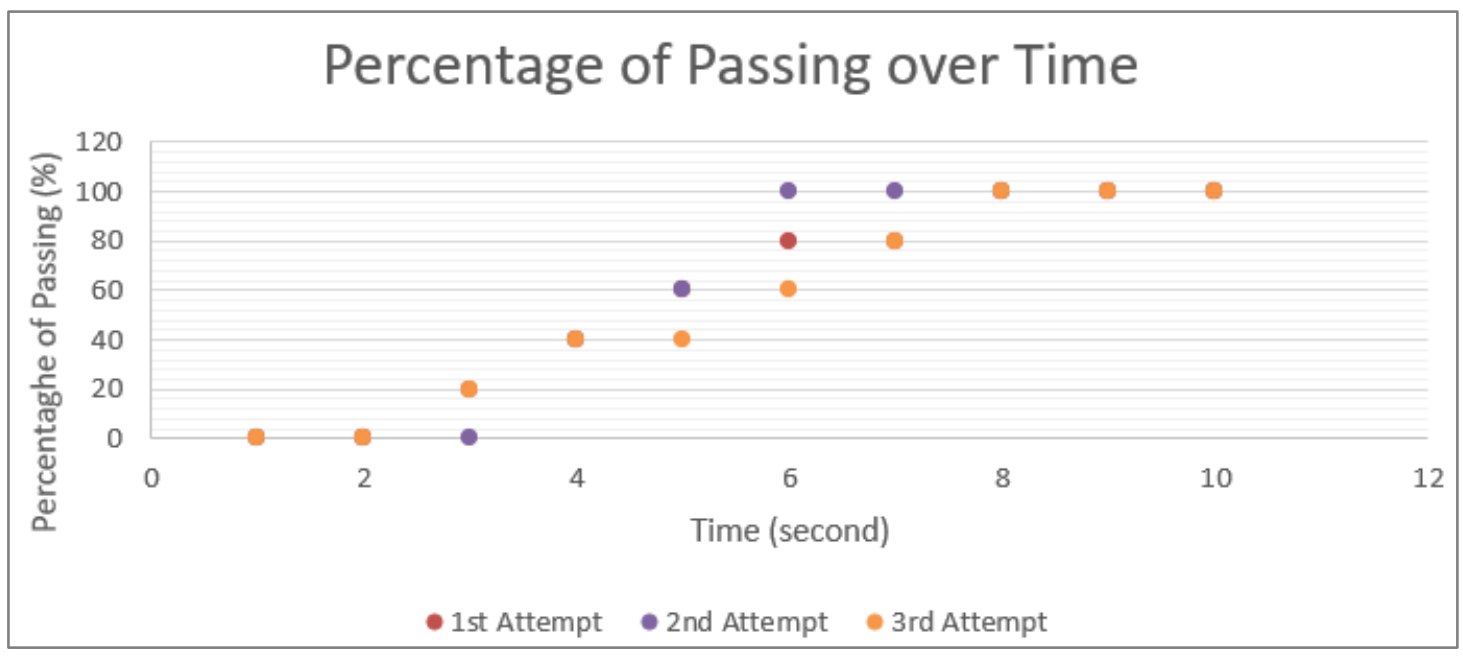

Figure 10. Graph of Percentage Passing over Time

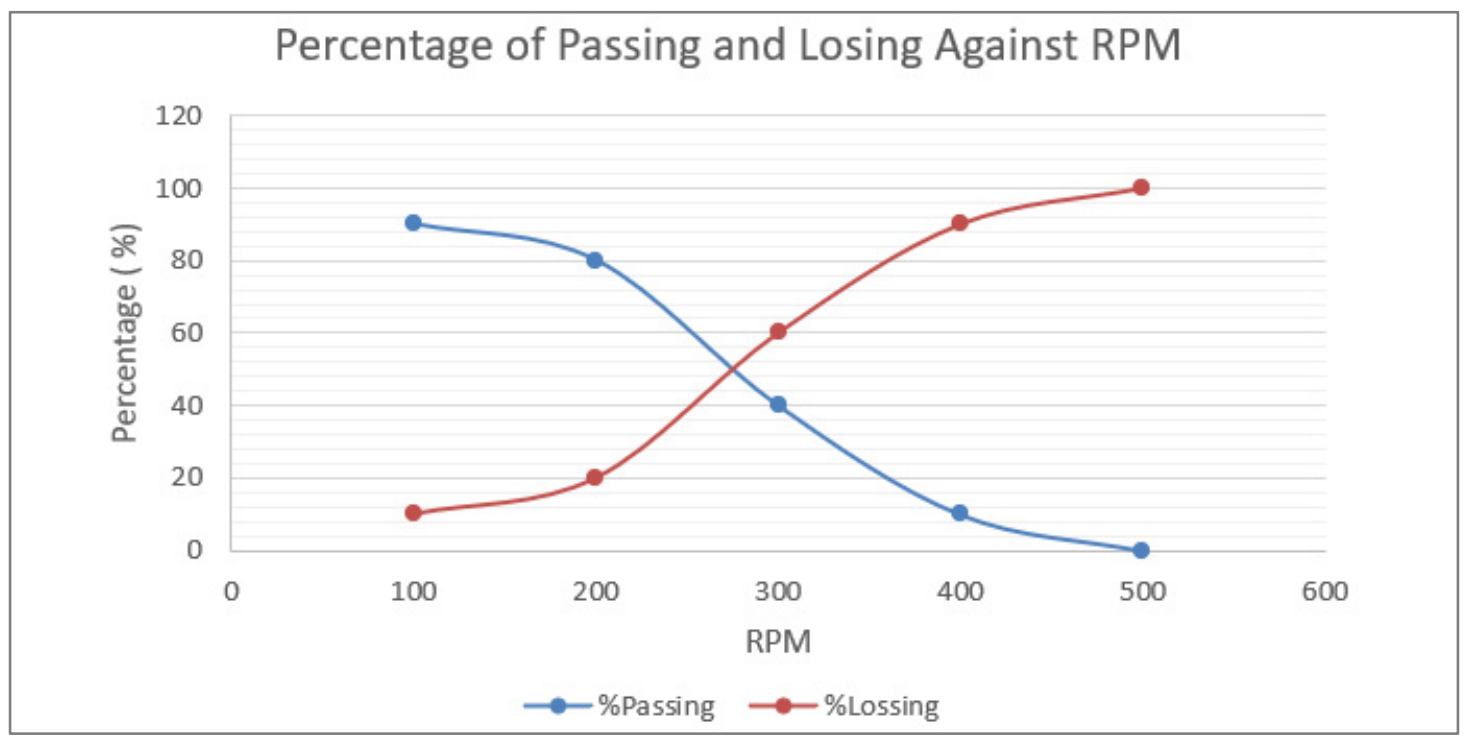

Figure 11. Graph of Percentage Passing and Losing against RPM 


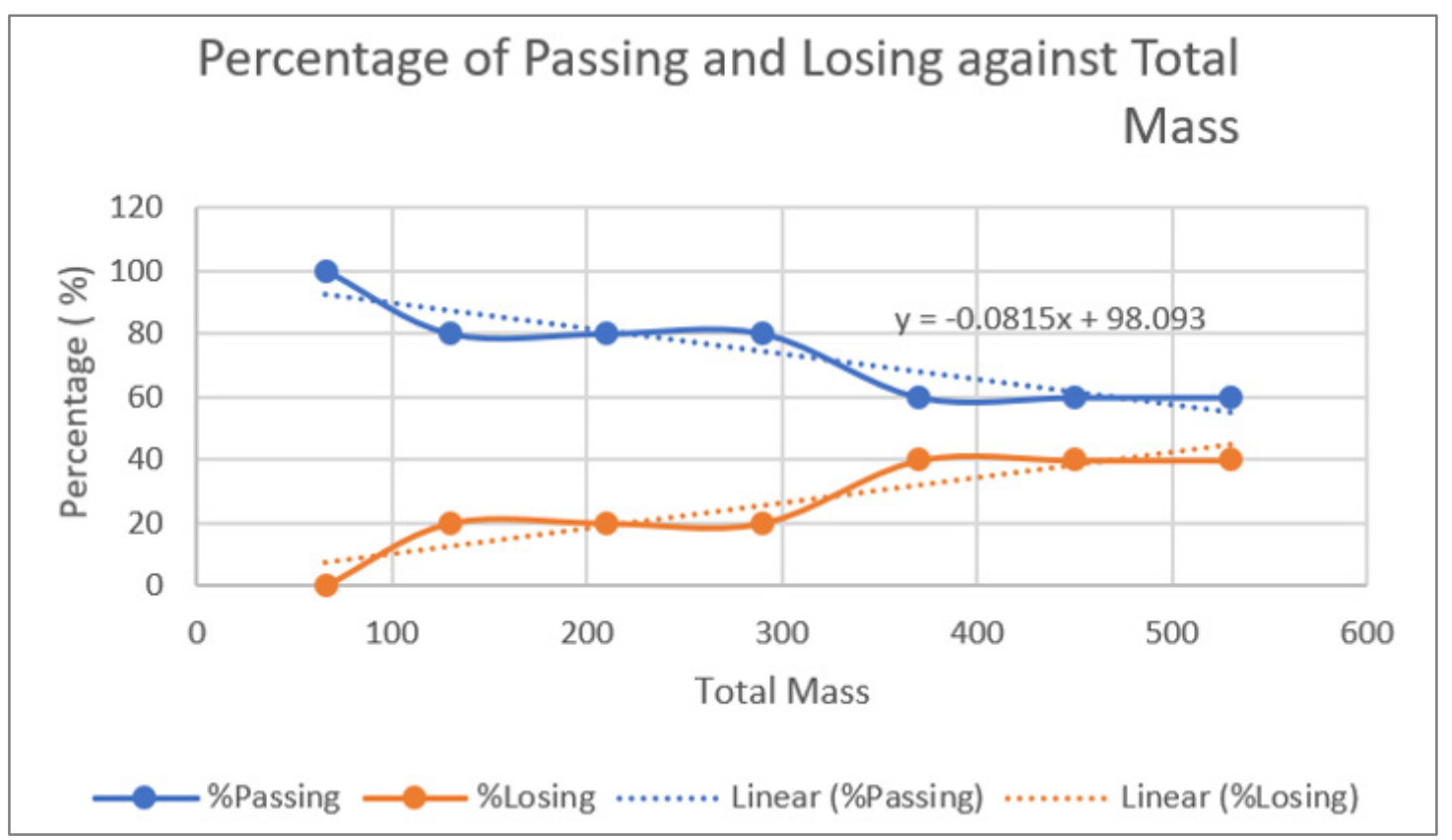

Figure 12. Graph of Percentage Passing and Losing against Total Mass

The slopes from Figure 11 indicate an increasing percentage of losing and decreasing percentage passing over RPM. This circumstance shows that most of the sample materials bypass the screen in the sieving process at a higher rotation speed. The optimum rotation speed for this test is acceptable at the range less than 100 RPM as higher RPM than that value cause exceeding thrust on ball bearings before the ball bearings can pass through the screen by the pulling of gravity, they were thrown at almost horizontal motion and bypass the screen. This problem can be solved by increasing the slope of the mesh surface, as the uphill action required a higher thrust of the centrifugal action.

\subsection{Determination of the Critical Ratio Coefficient}

In this case, the sample mass is maintained at a fixed amount, which is $50 \mathrm{~g}$ equivalents to 5 small ball bearings in this test. Under constant RPM, the percentage passing and losing shall meet a critical point on the slope. The maximum volume of the container for a mesh surface elevation of $30^{\circ}$ can be computed from the geometry calculation, which is about $2000 \mathrm{~cm}^{3}$ in this test. The problem of the test if the volume of the samples is difficult to be determined, so the assumption is made that each material has the same density, $8 \mathrm{~g} / \mathrm{cm}^{3}$. Another problem is that the test samples amount is not enough to fill the entire geometry, so the results beyond 30 large ball bearings equivalents to $480 \mathrm{~g}$ were unable to be determined. The graph of percentage passing and losing against total mass is shown in Figure 12.

From the graphs, the percentage passing is at its highest at least total mass, which indicates that the assumption is proven, the slope is decreasing against greater mass or volume. The critical ratio coefficient can only be roughly predicted with linear regression of the data, which is around $1200 \mathrm{~g}$ equivalent to $150 \mathrm{~cm}^{3}$. This value had denied the assumptions as the ratio to total volume is considered very low. A possible explanation for this might be that the effect of smooth and round properties of ball bearings has minimum resistance that will not throttle the sieving efficiency at all.

\section{Conclusions}

This study designed and developed a size segregation sieve machine using centrifugal action in the impurity's separation process. Findings from the study show that the machine is useful in the impurities separation process if high RPM is achieved. The most significant design considerations are the rotational speed of the machine. It could be achieved by using a higher power motor with more stable mounting to prevent unnecessary vibrations due to the high-speed motor. The series of tests were conducted by using ball bearings for ease of calculations and noticeability. In conclusion, this machine is useful for particle segregation applications and other potential applications such as soil separation. For future study, it is recommended to improve the current design in terms of parts and size simplification and multiple size segregation adaption for a higher production rate.

\section{REFERENCES}

[1] Abdullah, N. (2013). The Oil Palm Wastes in Malaysia. In F. 
S. E.-M. D. Matovic (Ed.) (p. Ch. 3). Rijeka: IntechOpen. https://doi.org/10.5772/55302

[2] Colombo, C., \& Vergani, L. (2018). Optimization of filament winding parameters for the design of a composite pipe. Composites Part B: Engineering, 148(March), 207 216. https://doi.org/10.1016/j.compositesb.2018.04.056

[3] Elamin, K. E. (1981). Determination of the design and operating parameters of rotary cleaning sieves for combines.

[4] Lee, M. D., Osman, M. S., Wong, G. K., \& Ngu, H. J. (2018). Chemical Oxygen Demand Reduction in Palm Oil Mill Effluent Treatment with Chitosan and Ferric Chloride. Advanced Journal of Technical and Vocational Education, 2(2), 19-23. https://doi.org/10.26666/rmp.ajtve.2018.2.3

[5] Leschonski, K. (1979). Sieve Analysis, the Cinderella of particle size analysis methods? 24, 115-124.

[6] Liew, W. L., Kassim, M. A., Muda, K., Loh, S. K., \& Affam, A. C. (2014). Conventional methods and emerging wastewater polishing technologies for palm oil mill effluent treatment: A review. Journal of Environmental Management, 149, 222-235. https://doi.org/10.1016/j.jenvman.2014.10.0 16
[7] Liu, K. (2009). Some factors affecting sieving performance and ef fi ciency. Powder Technology, 193(2), 208-213. https://doi.org/10.1016/j.powtec.2009.03.027

[8] Rosli, F., Mohd, C., Ghazali, R., Mustafa, M., Bakri, A., \& Hussin, K. (2016). A Review: Characteristics of Oil Palm Trunk (OPT) and Quality Improvement of Palm Trunk Plywood by Resin, 11, 5565-5580.

[9] Sandberg, D., Kutnar, A., \& Mantanis, G. (2017). Wood modification technologies - A review. IForest, 10(6), 895908. https://doi.org/10.3832/ifor2380-010

[10] Sukiran, M. A., Abnisa, F., Wan Daud, W. M. A., Abu Bakar, N., \& Loh, S. K. (2017). A review of torrefaction of oil palm solid wastes for biofuel production. Energy Conversion and Management, 149, 101-120.https://doi.org/10.1016/j.encon man.2017.07.011

[11] Zainal, N. H., Jalani, N. F., Mamat, R., \& Astimar, A. A. (2017). A review on the development of palm oil mill effluent (POME) final discharge polishing treatments. Journal of Oil Palm Research, 29(4), 528-540. https://doi.org/10.21894/jopr.2017.00012 